\title{
Measurements from a novel interferometer for EUVL mirror substrates
}

\author{
Max L. Krieg ${ }^{* a}$ and Joseph J. M. Braat ${ }^{\mathrm{a}}$ \\ ${ }^{a}$ Faculty of Applied Sciences, Delft University of Technology, Lorentzweg 1, 2628 CJ, Netherlands.
}

\begin{abstract}
A previously reported interferometer without intermediate optics is used to perform measurements on an aspherical extreme ultraviolet lithography mirror substrate. Acousto-optic modulation based phase shifting is used together with a novel phase retrieval algorithm to retrieve the phase distribution from our interferograms. The phase distribution is then processed by a previously reported inverse propagation algorithm to give the shape of the mirror under test. Our results are compared with measurements performed with conventional Fizeau interferometry and the discrepancies are discussed with reference to systematic error sources inherent in the classical and novel interferometers.
\end{abstract}

Keywords: interferometry, EUVL, phase shifting, metrology.

\section{INTRODUCTION}

The accurate measurement of the low spatial frequencies of aspheric reflector substrates for extreme ultraviolet lithography (EUVL) requires the use of novel metrology techniques. An interferometer developed at the Delft University of Technology circumvents the problematic issues associated with the need for accurate reference optics by instead using the wavefront from a single-mode optical fiber as a virtual reference surface. The instrument measures the optical path difference (OPD) arising from interference between such a reference wavefront, and an identically generated wavefront aberrated by reflection off the substrate under test. This optical path difference can be used to reconstruct the wavefront reflected from the substrate at the measurement plane. Relating this wavefront back to the substrate's shape is an exercise in inverse propagation, discussed in a previous publication ${ }^{1}$. The target accuracy of the instrument, using a metrology wavelength of $633 \mathrm{~nm}$, is $0.1 \mathrm{~nm}$. We will start by reviewing the working principle of the interferometer, before presenting data obtained with such an instrument. The interpretation of this data to give measurements of the substrate's shape will be described, followed by a comparison of the measurements with earlier measurements using a different detector and measurements using conventional Fizeau interferometry. The observed discrepancies will be discussed with reference to systematic error sources inherent in the classical and novel interferometers.

\section{THE INTERFEROMETER}

The details of the novel interferometer have previously been reported elsewhere ${ }^{1-8}$ but a brief review of the working principle will be presented for completeness.

Figure 1 shows a schematic diagram of the interferometer. Two fibers serve as point sources, providing wavefronts that can be considered spherical within the measurement accuracy ${ }^{9}{ }^{10}$. Light from the object fiber first falls onto the mirror substrate where it is reflected, passing through a focus and finally interferes with light from the reference fiber at the detection plane. To minimize the OPD gradients, and hence the fringe density, over the detection plane, the fiber tips should lie on a plane parallel to the detection plane and the focus should occur half way between the two fiber tips. This requires that the mirror substrate be placed approximately a distance R, equal to the substrate's radius of best fit, away from the fiber tips. The detector array should be placed at a distance away from the focus such that the resulting illumination just covers the array. For typical mirror substrates ${ }^{11}$, the fringe density can exceed the pixel density, and multiple wavelength interferometry can be used to retrieve the absolute OPD in the face of the significant undersampling of the fringes ${ }^{2}$.

"m.1.krieg@tnw.tudelft.nl; phone: +31 15278 2455; fax: +31 152788105 


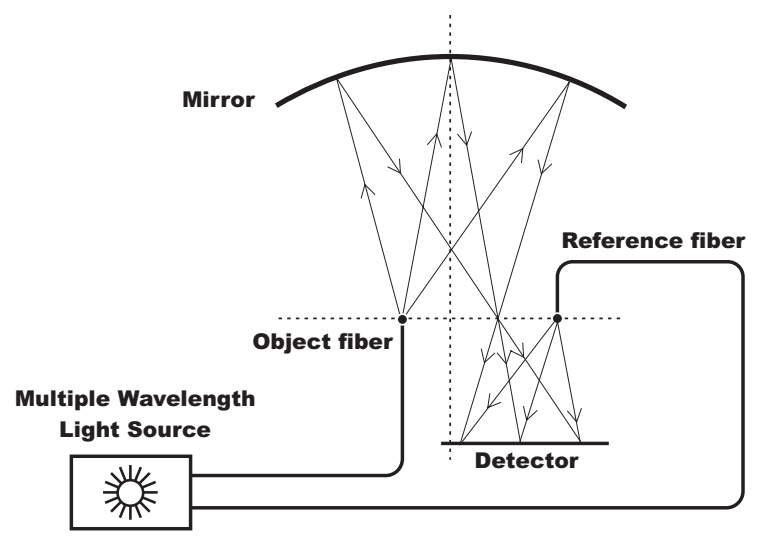

Figure 1: Schematic interferometer setup.

To recover the required range of spatial frequencies for the substrate (not for the fringe-pattern), the detector array must contain at least $25 \times 25$ sensors. The available lightsource- and detection- systems permit the use of both phase-shifting interferometry or heterodyne interferometry at much higher resolutions than this.

For the results reported in this paper, phase-shifting interferometry techniques were used to obtain the phase distribution of the interferogram at the detector. Two Acousto-optic modulators (AOMs) were used to up-shift the frequency of the light through the reference- and object-arm by the same amount, but allowing a relative phase-shift to be introduced between them.

The resulting interferograms were then recorded by a full-frame sensor, the frame-rate of which was synchronized to the phase-shifts. The phase difference between successive frames was typically $\pi / 2$ radians, and a total of 32 frames were recorded for each measurement, at a frame-rate of $24 \mathrm{fps}$.

Initial measurements using an old Sony CCD (XC-77RR-CE), showed artifacts which may have arisen as a result of the cover-glass bonded to the CCD housing. After attempts to remove the cover-glass without damaging the CCD proved unsuccessful, the experiments were repeated with a Kodak CCD sensor (KAI-2020) which not only had a greater resolution than the Sony CCD, but was also manufactured without the usual cover-glass bonded to the housing. Calibration measurements with the Kodak sensor proved to be much more accurate.

\section{PHASE RETRIEVAL}

In order to make a meaningful estimate of the optical phase difference between the reference and object wavefronts at each pixel, we will assume that the instantaneous light intensity $\left(I_{j, k}\right)$ at pixel number ' $j$ ' and frame index ' $k$ ' is given by:

$$
I_{j, k}=O_{j}+A_{j} \cos \left[k \omega+\phi_{j}\right]+n_{j, k}
$$

Where ' $O_{j}$ ' is the offset, ' $A_{j}$ ' is the amplitude and ' $\phi_{j}^{\prime}$ ' is the phase of the interference pattern at pixel ' $j$ '. The phase-step ' $\omega$ ' is assumed to be constant, both over time and over all pixels, while the noise, ' $n_{j, k}$ ' is assumed to be a stochastic quantity.

If the noise-process in our instantaneous intensity measurements at each pixel has a Gaussian distribution (a reasonable assumption), we can find the maximum likelihood estimators (MLE) for the amplitude, offset and phase of the signal at each pixel, as well as the global phase-step, by minimizing the sum of squared differences between the measured data and the predicted data over the aforementioned parameters.

This 'misfit' function is shown in (2), re-written in a form where the phase-offset cosine of (1) is replaced with the sum of a sine and cosine of variable amplitudes. The number of fitting parameters remains the same, but now all parameters, save for the global phase-step, are linear and their optimal value can therefore be found analytically, provided that more than three frames have been measured. 


$$
\begin{aligned}
& M\left(\phi_{j}, A_{j}, O_{j}, \omega\right)=\sum_{j=1}^{M} \sum_{k=1}^{N}\left(I_{j, k}-a_{j} \sin [k \omega]-b_{j} \cos [k \omega]-O_{j}\right)^{2} \\
& \phi_{j}=\tan ^{-1}\left(a_{j} / b_{j}\right), \quad A_{j}=\sqrt{a_{j}^{2}+b_{j}^{2}}
\end{aligned}
$$

Here, $M$ is the misfit function of the amplitude, offset and phase of the signal at each pixel, as well as the global phasestep. The parameters ' $a$ ' and ' $b$ ' are simply convenient transforms of $\phi$ and $A$, to make the misfit function linear and therefore allow analytical minimization over these parameters. The index $j$, denotes the pixel number and runs from 1 to the number of pixels considered (M), whereas the index $k$, denotes the frame index, or phase-step number, and runs from 1 to the number of frames taken $(\mathrm{N})$.

The minimization over the global phase-step parameter requires an iterative numerical procedure. This can be performed in a reasonable time on a modern computer, yielding an optimal estimate of the parameters in a statistical sense. To speed up the process at the cost of some accuracy, a subset of pixels can first be chosen for which the optimal phase-step is determined. This phase-step can then be used to determine the remaining parameters for all pixels analytically.

This approach yields the best possible estimate of the phase, amplitude and offset at each pixel of our measurements (in a statistical sense, provided the noise process is Gaussian).

Unfortunately, this process cannot correct for systematic errors such as cross-talk between the object and reference arms, or non-uniform phase-steps. The histogram correction described in the thesis on this topic ${ }^{5}$ is used here to reduce these effects.

\section{PHASE UNWRAPPING}

From initial estimates of the interferometer parameters and mirror shape, we calculate the theoretical absolute optical path difference at each pixel. This leads to a theoretical fringe pattern which we subtract from our observed pattern. The resulting difference $(\bmod 2 \pi$ ) shows a much smaller number of phase-jumps and can be unwrapped line-by-line after masking any low fringe-contrast areas in our interferogram (Figure 7 \& Figure 8). To prevent phase-jumps between lines, an integer number multiple of $2 \pi$ is added to every connected line segment in such a way as to minimize the difference between this segment and the previous line.

After un-wrapping, we can add the absolute optical path difference previously subtracted back to our un-wrapped interferogram to give the measured absolute optical path difference which will be used to determine the mirror shape.

\section{SENSOR CALIBRATION}

It is assumed that sensors will first have to be calibrated for errors in their pixel positions. Warping of CCD silicon substrates is a well documented phenomenon, which could give rise to a shift in the position of pixels with respect to each other. While out-of-plane warping is likely to be more extensive than in-plane warping, especially after any bonding of the wafer to its housing, the latter will nonetheless be more significant, as the interferometer is more sensitive to in-plane errors.

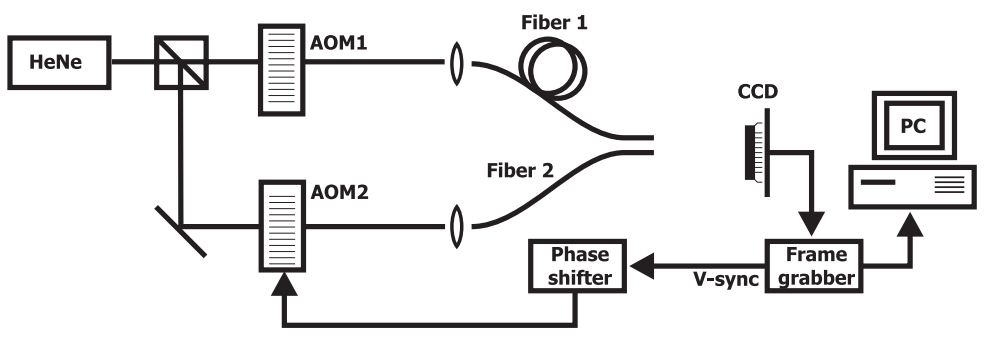

Figure 2: Two-fiber calibration setup for CCD sensor. 
In order to calibrate the position of each pixel relative to the other pixels, a simplified calibration setup with only two fibers is used (see Figure 2). If a significant phase-gradient is introduced, the measured phase is equal to the phase at the nominal pixel position, plus an error term proportional to the phase gradient and the pixel position error in the direction of this gradient:

$$
O P D(x, y) \approx O P D\left(x_{n}, y_{n}\right)+\frac{\partial O P D}{\partial x}\left(x-x_{n}\right)+\frac{\partial O P D}{\partial y}\left(y-y_{n}\right)
$$

The theoretical optical path difference (OPD) is easily modeled, since the fiber tips may be considered point sources. If we are to assume that any differences between the measured and theoretical OPDs are due solely to noise and pixelpositioning errors, we may take the OPD at the nominal pixel positions as the theoretical OPD at these positions.

The theoretical OPD is also a function of the fiber-tip positions and, while great care has been taken to measure these, our measurements are later further refined by fitting the theoretical OPD to the measured OPD in a least-squares sense, using the fiber-tip positions as our parameters. This optimization results in an improved estimate of the fiber positions, which should remain within the measurement accuracy of our original measurements.

In order to determine the pixel position error in two dimensions, two non-degenerate calibration measurements need to be carried out. Using two such measurements, we can solve for the actual pixel positions as follows:

$$
\begin{gathered}
x=x_{n}+\frac{\left(\frac{\partial O P D_{2}}{\partial y} \Delta O P D_{1}(x, y)-\frac{\partial O P D_{1}}{\partial y} \Delta O P D_{2}(x, y)\right)}{\left(\frac{\partial O P D_{2}}{\partial y} \frac{\partial O P D_{1}}{\partial x}-\frac{\partial O P D_{2}}{\partial x} \frac{\partial O P D_{1}}{\partial y}\right)} \\
y=y_{n}-\frac{\left(\frac{\partial O P D_{2}}{\partial x} \Delta O P D_{1}(x, y)-\frac{\partial O P D_{1}}{\partial x} \Delta O P D_{2}(x, y)\right)}{\left(\frac{\partial O P D_{2}}{\partial y} \frac{\partial O P D_{1}}{\partial x}-\frac{\partial O P D_{2}}{\partial x} \frac{\partial O P D_{1}}{\partial y}\right)}
\end{gathered}
$$

Where $\triangle O P D=O P D(x, y)-O P D\left(x_{n}, y_{n}\right)$ and the gradients are evaluated at $\left(x_{n}, y_{n}\right)$.
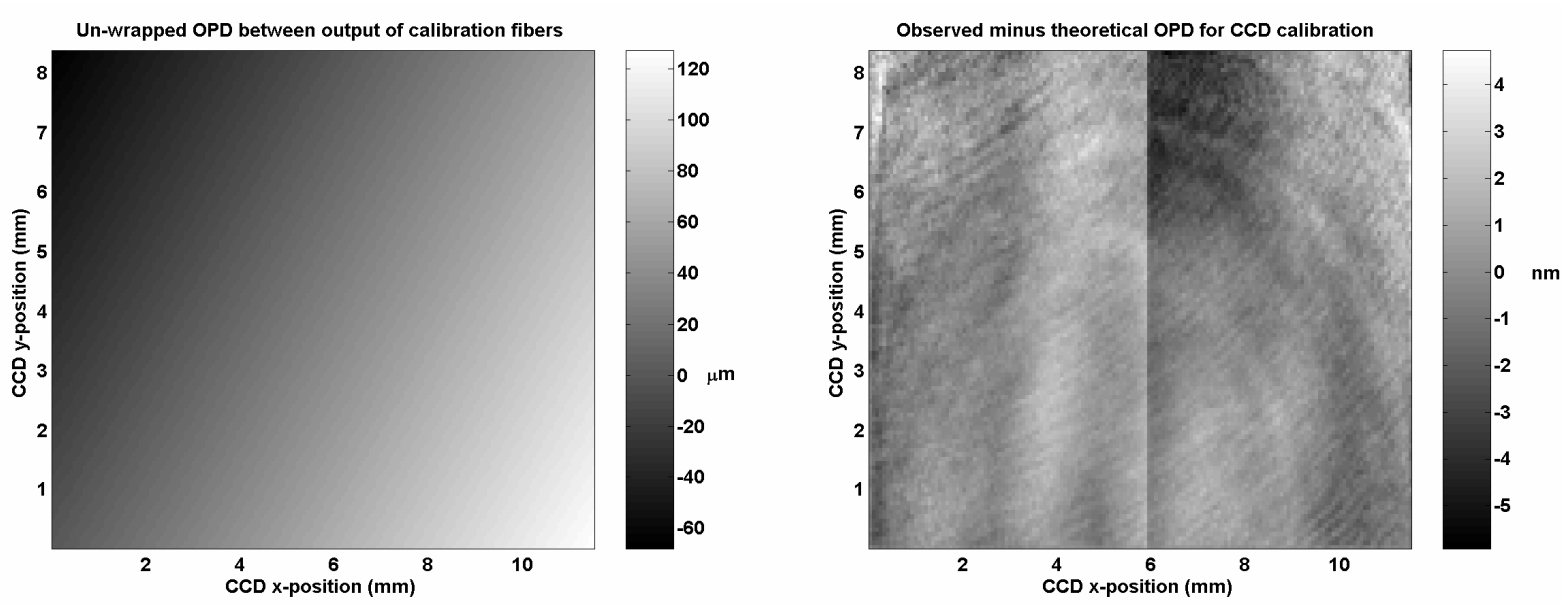

Figure 3: Observed optical phase difference (OPD, un-wrapped, left) and difference between observed and theoretical OPD (right) 
Figure 3 shows measured data from a calibration measurement on the Kodak sensor (un-wrapped) as well as the difference with the theoretical phase distribution arising from two spherical wavefronts, originating at $(5.558,-1.989,59.378) \mathrm{mm}$ and $(6.215,-2.495,59.335) \mathrm{mm}$ from the bottom-left corner of the CCD respectively. Of note should be the discontinuity along the middle of the CCD, along with the low spatial frequency components. Together with a second measurement with different relative fiber positions, a "pixel displacement map" can be generated (see Figure 4). The largest absolute displacement measured was $0.6 \mu \mathrm{m}$, compared to a pixel spacing of $7.4 \mu \mathrm{m}$. The rms pixel-to-pixel deviation is merely $30 \mathrm{~nm}$. It should be noted that the displacement map has been convolved with a gaussian kernel with a 4-pixel standard deviation in order to reduce the effects of noise. The implicit assumption is made that warping will not introduce high spatial frequency pixel positioning errors.

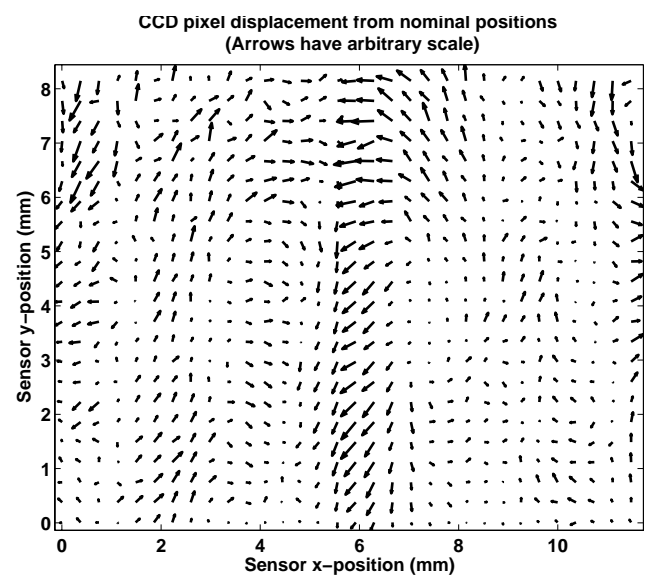

Figure 4: Quiver plot of pixel displacement from nominal positions (vectors are severely exaggerated for clarity)

With the calibration of the actual pixel positions, we are now able to interpret interferograms of mirror measurements.

\section{BACKPROPAGATION}

The back-propagation algorithm described elsewhere ${ }^{1,5}$ is very sensitive to the so-called "interferometer parameters" the relative positions of the fibers and CCD and the distance of the mirror-apex to the object fiber. Since the measurements presented here were made before the interferometer parameters could be properly calibrated, they will need to be fitted. This fitting can only introduce a small class of figure-errors in our final measurements as described in the thesis ${ }^{5}$, but it should nonetheless be noted that this step introduces a bias towards the nominal mirror shape.

The treatment presented here does not make use of the diffraction compensation algorithm described ${ }^{1,5}$, but instead relies on the spatial filtering of a 42-term Zernike decomposition to reduce the diffraction effects observed. Apart from additional computation effort, there is no obstacle to applying the full diffraction compensation algorithm to the data in the future. 


\section{DATA}

Figure 5 shows a measurement of the figure errors of our mirror substrate, carried out by independent, state of the art, self-calibrated Fizeau interferometer measurements. While the absolute accuracy of these measurements cannot be confirmed, the measurement will serve as a point of comparison with our measurements.
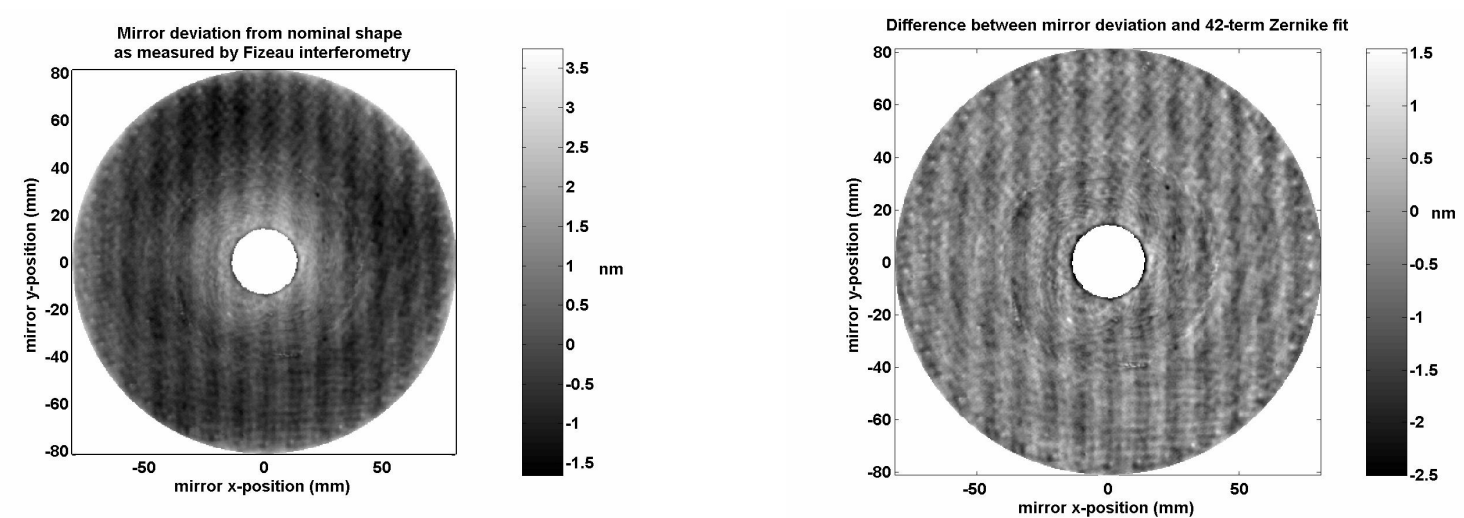

Figure 5: Figure errors of test mirror as measured by independent, self-calibrated Fizeau interferometry, along with the remainder after subtracting a 42-term Zernike decomposition of the data.

Figure 6 shows a typical fringe-pattern from our interferometer using the Kodak KAI-2020 CCD.
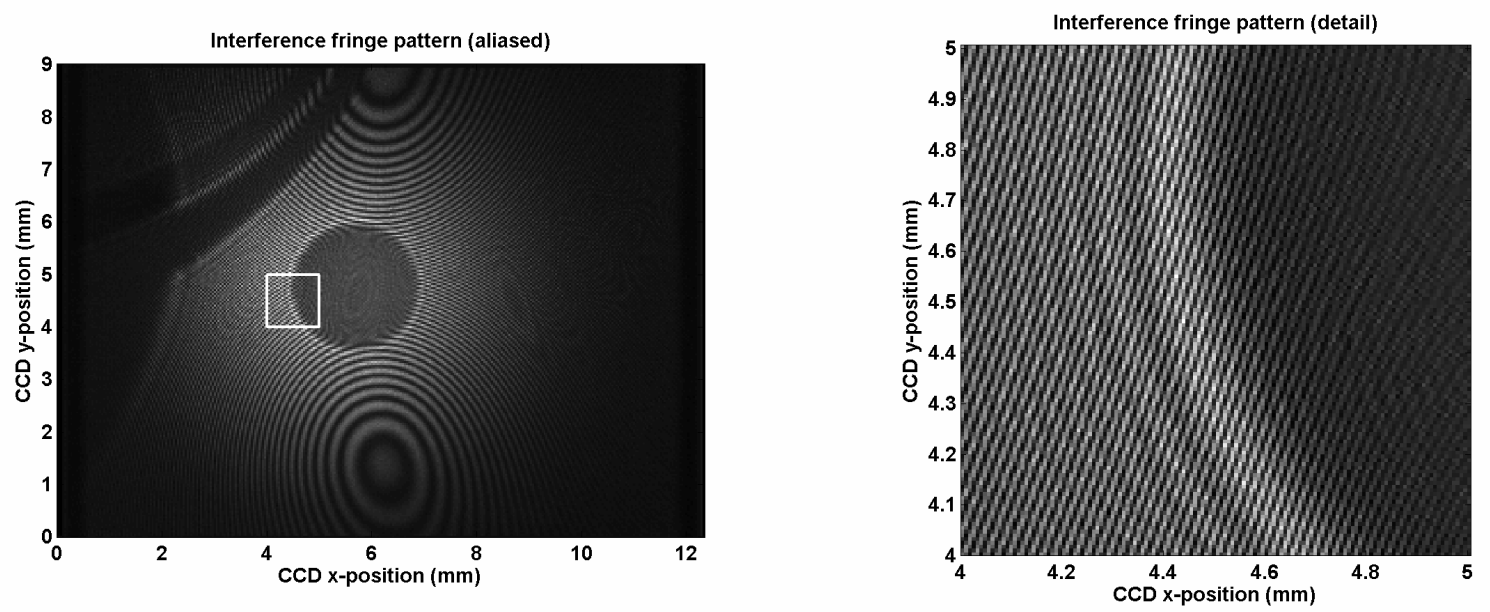

Figure 6: Intensity interference fringe pattern on Kodak CCD, including detail showing high fringe frequency. The features visible in the top-left corner of the full pattern are fibers obstructing the beam.

Subtracting a theoretical phase distribution, using the nominal mirror shape and interferometer parameters, from the measured phase results in the pattern seen in Figure 7. The un-wrapping procedure previously described results in the OPD map shown in Figure 8. Regions which contain low contrast or obstructions are masked, and are not considered during further calculations. This data (using the calibrated relative pixel positions) is then decomposed into the first 42 best-fitting Zernike functions before being inverted using the back-propagation algorithm previously described ${ }^{1,5}$. Use of the nominal interferometer parameters results in an excessive figure-error (Figure 9) and parameter fitting was employed to estimate the correct interferometer parameters. Figure 9 also shows the figure error calculated using the fitted interferometer parameters. 


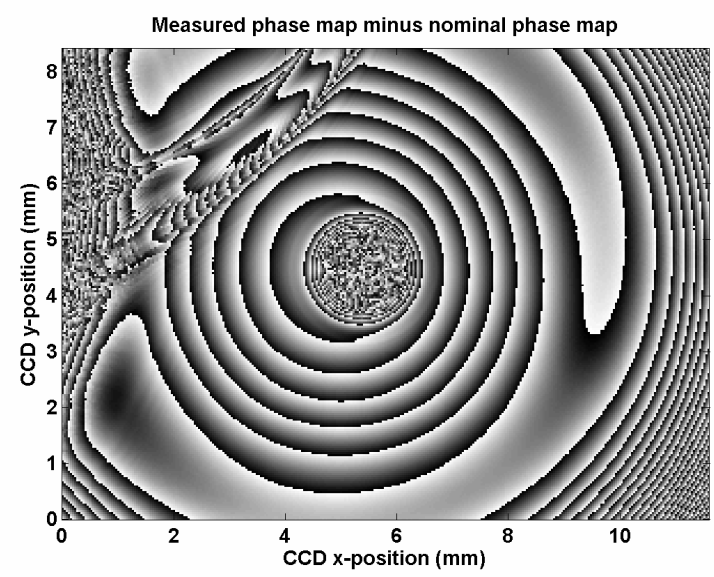

Figure 7: Difference between the measured phase map and the phase map calculated from the nominal mirror shape (mod $2 \pi)$. Note again the presence of the obstructing fibers.
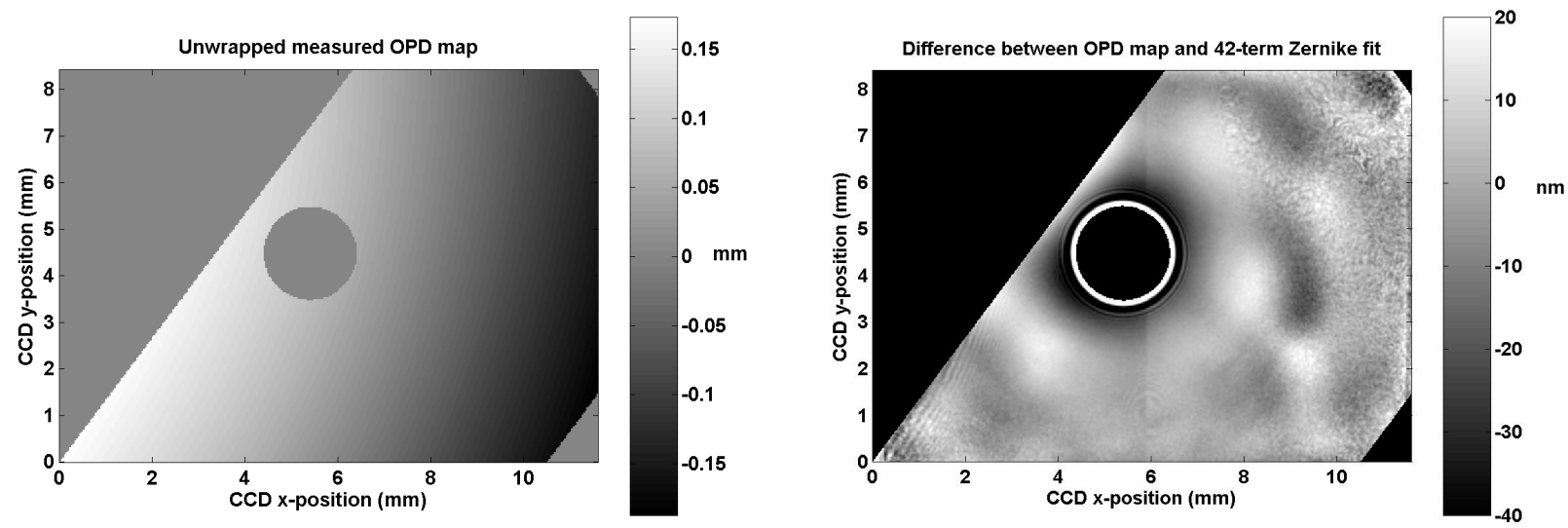

Figure 8: Optical path difference map from measured data, along with the remainder of this data after subtracting a 42-term Zernike polynomial fit thereto. Regions of bad fringe contrast are masked manually here.
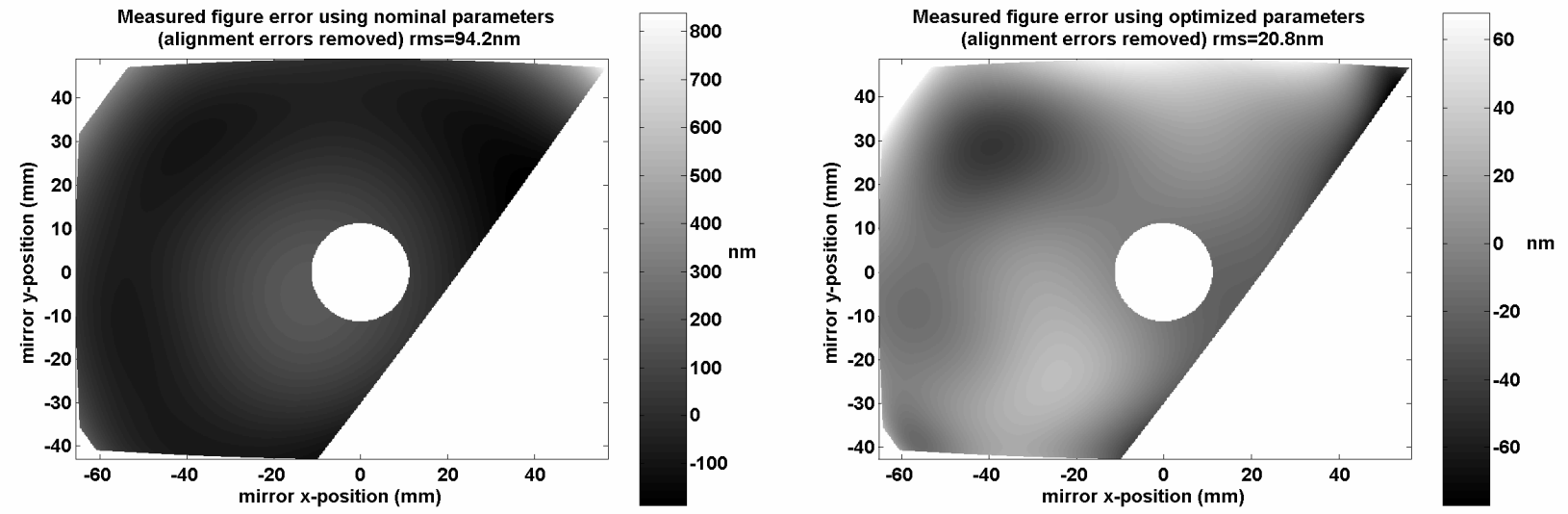

Figure 9: The figure errors calculated from the 42-term Zernike fit to our data, using the nominal (left) and optimized interferometer parameters (right). 
Similar figures for the measurements carried out with the Sony CCD are shown in Figures 10-12. It is important to note that the Sony CCD has a much smaller field of view, measuring only about $23 \mathrm{~mm}$ around the center of this mirror, as opposed to the $\sim 60 \mathrm{~mm}$ that can be measured with the Kodak CCD.
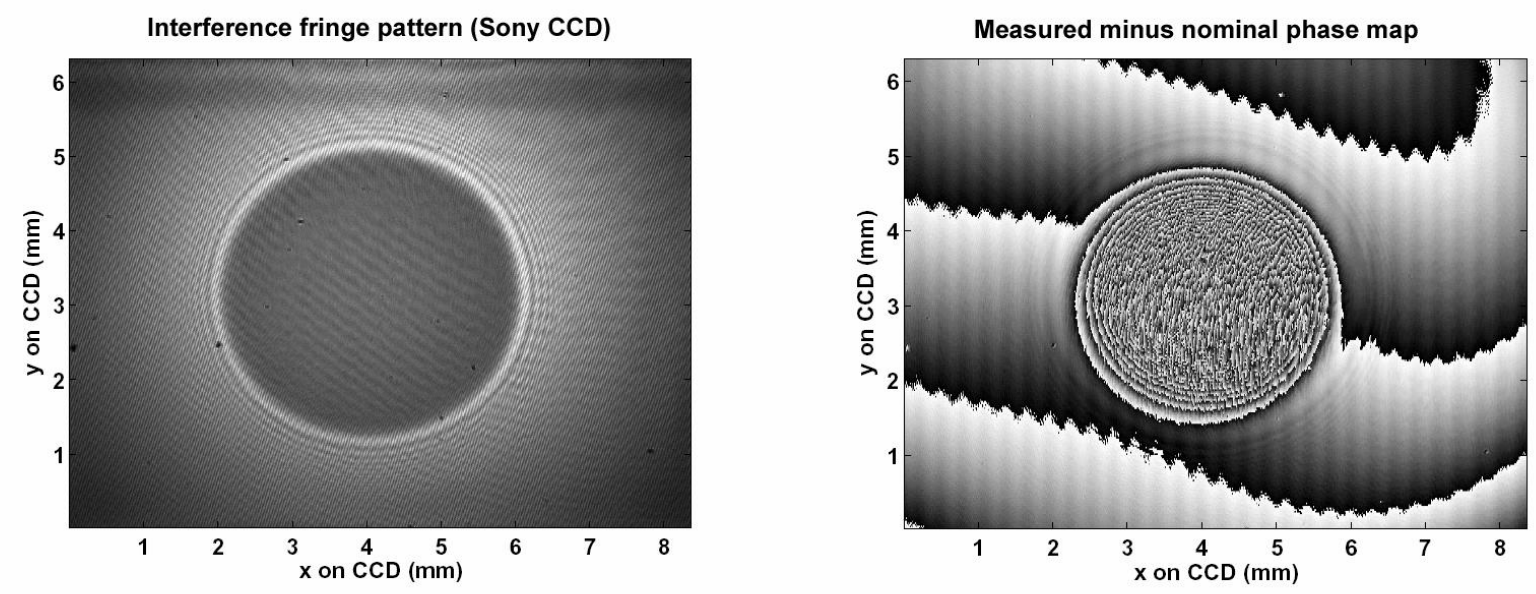

Figure 10: Intensity interference fringe pattern (left) on Sony CCD, along with mod $2 \pi$ difference between measured phase map and phase map calculated from nominal mirror shape (right).
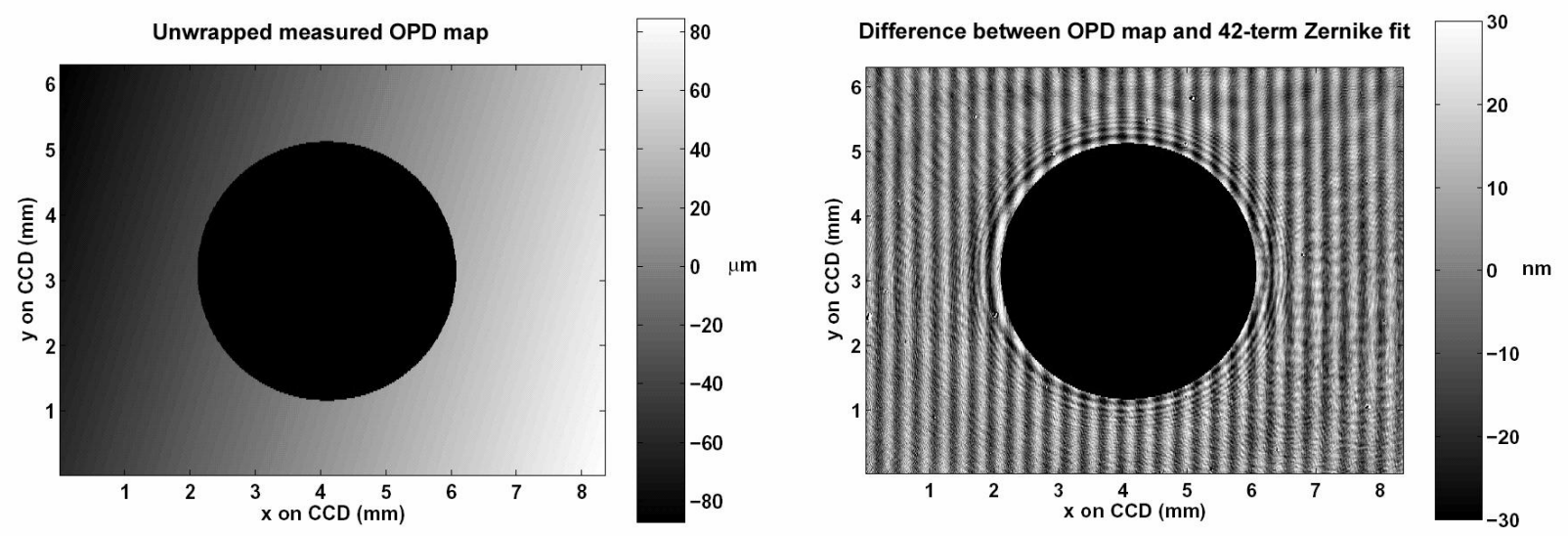

Figure 11: Optical path difference map from measured data, along with the remainder of this data after subtracting a 42-term Zernike polynomial fit thereto. Regions of bad fringe contrast are masked manually. 


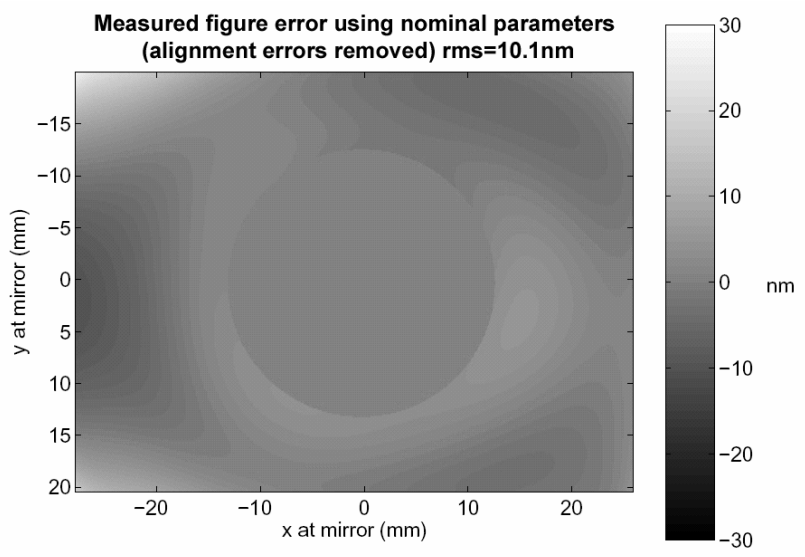

Figure 12: Figure errors calculated from the 42-term Zernike fit to the Sony data, using nominal interferometer parameters. The optimized parameters reduce the rms value to $4.2 \mathrm{~nm}$, but do not significantly alter the form of the error map.

\section{ANALYSIS}

Unfortunately, there are a large number of discrepancies between these three measurements. From the difference between the measured and predicted phase maps for the Kodak measurements (Figure 7), we can conclude that there is a significant amount of defocus present, most likely arising from bad alignment of the interferometer. This is also evident from the obstruction observed. The interferometer is designed to let the beams pass unobstructed with proper alignment. While the parameter optimization can go some way towards estimating the degree of this misalignment, there are too many parameters, some of which are almost degenerate, to perform a global optimization in a reasonable amount of time. The rms figure error of $20.8 \mathrm{~nm}$ for the optimized parameters, shown in Figure 9, is excessive considering the independent Fizeau measurement of Figure 5.

While the Kodak measurements do not have the required accuracy to measure the mirror shape, due to bad alignment of the interferometer in this instance, they would have the required sensitivity to pick up features such as the horizontal stripes observed in both the Fizeau and Sony measurements (Figure 5 \& Figure 11). The fact that no stripes are observed in Figure 8 may indicate that the stripes in the other measurements are artifacts of the corresponding measurement methods. This is further supported by the observation that the stripes of the Sony and Fizeau measurements to not correspond to each other, having a much higher spatial frequency in the Sony measurements. While the CCD cover glass is a likely suspect for the cause of the stripes in the Sony measurements ${ }^{5}$, not enough is known about the components used in the independent Fizeau measurements to identify a likely cause. Unlike these two measurements, the Kodak measurements used absolutely no optical elements which could have introduced such artifacts.

One last point of interest is the remaining vertical discontinuity seen in Figure 8, at the same location as the discontinuity observed in the sensor calibration measurements (Figure 4). In the calibration experiment, the two fibers were not placed at the same position as the reference fiber and focus in the actual experiment. For different angles of incidence of light, pixels may well exhibit a slightly different effective detection location. Repeating the calibration experiment with fiber positions more closely matching the reference fiber and focus locations of the actual experiment may therefore reduce this last artifact.

\section{CONCLUSION}

The sensitivity of our novel interferometer is sufficient to determine that vertical artifacts seen in other measurements are likely to be due to the measurement method rather than the object under test. However, without more careful alignment procedures and measurements of the interferometer parameters, our interferometer is unable to reach the accuracies required by our application. Once this has been achieved, some further improvements may be achieved by fine-tuning the sensor calibration procedures used. 


\section{ACKNOWLEDGEMENTS}

This work was supported by the Dutch Technology Foundation STW, by ASM Lithography, Veldhoven, The Netherlands, and by Carl Zeiss, Oberkochen, Germany.

\section{REFERENCES}

1. M. L. Krieg and J. J. M. Braat, "Inverse propagation algorithm for angstrom accuracy interferometer," in Interferometry XII: Techniques and Analysis, vol. 5531, Proceedings of the Society of Photo-Optical Instrumentation Engineers (SPIE), K. Creath and J. Schmit, Eds., 2004.

2. R. G. Klaver, "Novel interferometer to measure the figure of strongly aspherical mirrors." Delft: Delft University of Technology, 2001.

3. R. G. Klaver and J. J. M. Braat, "Novel interferometer to measure the figure of aspherical mirrors as used in EUV lithography," in Emerging Lithographic Technologies IV, vol. 3997, Proceedings of the Society of Photo-Optical Instrumentation Engineers (SPIE), 2000, pp. 784-793.

4. R. G. Klaver, H. van Brug, and J. J. M. Braat, "Interferometer to measure the form figure of aspherical mirrors as used in EUV lithography," in Laser Metrology and Inspection, vol. 3823, Proceedings of the Society of PhotoOptical Instrumentation Engineers (SPIE), 1999, pp. 123-132.

5. M. L. Krieg, "Absolute Heterodyne Interferometer for Strongly Aspherical Mirrors." Delft: Delft University of Technology, 2004.

6. M. L. Krieg, R. G. Klaver, and J. J. M. Braat, "Absolute optical path difference measurement with angstrom accuracy over ranges of millimetres," in Optical Measurement Systems for Industrial Inspection II: Application in Industrial Design, vol. 4398, Proceedings of the Society of Photo-Optical Instrumentation Engineers (SPIE), 2001, pp. 116-126.

7. M. L. Krieg, G. Parikesit, and J. J. M. Braat, "Three-wavelength laser light source for absolute, sub-Angstrom, two point source interferometer," in Optical Measurement Systems for Industrial Inspection III, vol. 5144, Proceedings of the Society of Photo-Optical Instrumentation Engineers (SPIE), 2003, pp. 227-233.

8. M. L. Krieg, B. L. Swinkels, and J. J. M. Braat, "Characterization of the frequency modulated continuous wave subsystem of an angstrom accuracy absolute interferometer," in Interferometry XI: Applications, vol. 4778, Proceedings of the Society of Photo-Optical Instrumentation Engineers (SPIE), 2002, pp. 131-141.

9. G. E. Sommargren, "Diffraction methods raise interferometer accuracy," Laser Focus World, vol. 32, pp. 61-\&, 1996.

10. G. E. Sommargren, "Phase shifting diffraction interferometry for measuring extreme ultraviolet optics," presented at Proceedings of Extreme Ultraviolet Lithography (ISBN 155752435 1). 1-3 May 1996 Boston, MA, USA [Opt. Soc. America], 1996.

11. T. E. Jewell, K. P. Thompson, and J. M. Rodgers, "Reflective Optical Designs for Soft X-ray Projection Lithography," vol. 3823, Proceedings of the Society of Photo-Optical Instrumentation Engineers (SPIE), 1991, pp. 123-132. 